\title{
Evaluation and management of patients referred to a tertiary-level hypertension clinic in Cape Town, South Africa
}

\author{
M S Moosa, ${ }^{1} \mathrm{MB}$ ChB, FCP (SA); L S Kuttschreuter, ${ }^{2} \mathrm{MB}$ ChB; B L Rayner, ${ }^{3} \mathrm{MB}$ ChB, FCP (SA), MMed, PhD \\ ${ }^{1}$ Department of Medicine, New Somerset Hospital, Cape Town, South Africa \\ ${ }^{2}$ Department of Medicine, Faculty of Health Sciences, University of Cape Town, South Africa \\ ${ }^{3}$ Division of Nephrology and Hypertension, Department of Medicine, Groote Schuur Hospital, Cape Town, South Africa
}

Corresponding author: M S Moosa (shiraz.moosa@gmail.com)

Background. Hypertension remains a global health burden, with a high incidence of long-term morbidity and mortality.

Objectives. To evaluate blood pressure (BP) control, factors associated with poor BP control, target organ damage (TOD), white-coat hypertension, treatment-resistant hypertension and secondary hypertension in patients referred to a tertiary-level hypertension clinic. Method. This was a prospective case-control study of patients referred for specialist hypertension management. Patient parameters recorded included age, gender, body mass index, uric acid, cholesterol, screening BP, follow-up BP, TOD and medications. We also recorded causes of secondary hypertension. Net BP change and the percentage achieving target BP were calculated in all patients followed up. Results. A total of 175 patients were sampled ( 72 males and 103 females, mean age 46.5 years). Of the patients $16.6 \%$ had a normal screening BP; $62.9 \%$ of patients were followed up, and $43.6 \%$ of these achieved BP control. After intervention, there was a net drop of $13.2 \mathrm{mmHg}$ (range 7.9 - 18.4) in systolic BP and of $3.8 \mathrm{mmHg}(4.4-12.0)$ in diastolic BP. Of all the patients, $12.6 \%$ had resistant hypertension, $49.1 \%$ had evidence of left ventricular hypertrophy and $18.3 \%$ had microalbuminuria; $13.1 \%$ of the patients were diagnosed with secondary hypertension.

Conclusion. Specialist intervention was useful in identifying patients with white-coat and secondary hypertension, as well as in improving hypertension control in patients with apparent treatment-resistant hypertension. However, a significant percentage of patients did not reach target BP, and further efforts are required to identify the underlying causes for this.

S Afr Med J 2016;106(8):797-800. DOI:10.7196/SAMJ.2016.v106i8.9610

Hypertension is a major risk factor for cardiovascular disease, and has been associated with two-thirds of strokes and almost half of ischaemic heart disease cases globally. ${ }^{[1]}$ High blood pressure (BP) was the foremost cause of death worldwide in 2008, responsible for $13-14 \%$ of global mortality or about 7.5 million deaths. ${ }^{[2]}$

In South Africa (SA) during the year 2000, 46888 deaths or 9\% of all deaths were attributable to this treatable condition. ${ }^{[3]}$ Hypertension is also a leading cause of heart failure and chronic kidney disease both locally and globally. Achieving optimal BP control in hypertensive patients remains an idealistic goal, with a population survey in SA in 1998 estimating control at 10\% for men and $18 \%$ for women. ${ }^{[4]}$

It appears that the burden of hypertension has increased exponentially over the past decade. A national household survey in 2010 (the National Income Dynamics Study) measured a prevalence of hypertension of $>40 \%$ in adults aged $>25$ years. ${ }^{[5]}$ Only $35.7 \%$ of individuals with hypertension were on treatment, and of these only $36.4 \%$ were controlled.

In another population survey (World Health Organization Study on Global Aging and Adult Health, 2007 ${ }^{[6]}$ ), compared with five other countries SA had the highest prevalence of hypertension $(77.9 \%)$ in individuals aged $>50$ years. Resistant hypertension is associated with poor outcomes and appears to be a particular problem in the uninsured population. ${ }^{[7]}$

There are few published data on the control of hypertension and the profiles of patients seen at tertiary-level hypertension clinics in SA. In a cohort derived from the Oslo Renal Denervation Study, 83 patients were referred to a tertiary-level hospital for work-up of apparent treatment-resistant hypertension; ${ }^{[8]}$ of these, 53 did not have true resistant hypertension, and the main reasons for this finding were poor drug adherence (32\%), secondary hypertension (30\%) and white-coat hypertension (15\%).

We therefore conducted a prospective study of patients referred to and followed up at a tertiary-level hypertension clinic at Groote Schuur Hospital, Cape Town, SA.

\section{Objectives}

To assess the following in all patients referred to our specialist hypertension clinic: (i) BP control and target organ damage (TOD); (ii) whether age, gender, body mass index (BMI), and uric acid and total cholesterol levels were independently associated with uncontrolled BP; and (iii) the prevalences of white-coat, apparent treatment-resistant and secondary hypertension.

\section{Methods}

The study was a 12-month prospective, descriptive, case-control study. It was conducted at Groote Schuur Hospital, a tertiarylevel academic hospital that provides services to a drainage area representing $51.9 \%$ of the Cape Town metro population. The study was approved by the Health Sciences Human Research Ethics Committee of the University of Cape Town (REC Ref. 220/2010). All patients referred for evaluation of apparent treatment-resistant hypertension were included in the analysis. Eligible patients were enrolled over a 1-year period beginning in August 2010. Data were collected at screening and until the last patient follow-up.

Patients were referred to the hypertension clinic from primary and secondary healthcare clinics in the hospital drainage area for evaluation of white-coat hypertension or apparent treatmentresistant hypertension. Patient data were collected at screening, and patients with abnormal automated office blood pressures 
(defined as a mean BP $>135 / 85 \mathrm{mmHg}^{[9]}$ ) were fully evaluated and followed up at the hypertension clinic. Patient data collected included age, gender, BMI, medications used and mean BP. We also checked serum potassium, creatinine, glucose, total cholesterol, renin and aldosterone and tested for the R563Q mutation of the epithelial sodium channel (ENaC). ${ }^{[10]}$ Left ventricular hypertrophy $(\mathrm{LVH})$ on the electrocardiograph (ECG) and an abnormal urinary albumin/creatinine ratio were used to assess TOD in all patients. LVH was defined using either the Sokolow-Lyon or the Cornell voltage criteria. A normal urinary albumin/creatinine ratio was defined as $<3 \mathrm{mg} / \mathrm{mmol}$, microalbuminuria as a urine albumin level of $3-30 \mathrm{mg} / \mathrm{mmol}$, and macroalbuminuria as $>30 \mathrm{mg} / \mathrm{mmol}^{\left[{ }^{[9]}\right.}$ The BMI and estimated glomerular filtration rate were calculated using the formula weight/height ${ }^{2}$ and the Modification of Diet in Renal Disease equation, respectively. ${ }^{[11]}$ Chronic kidney disease was defined according to the Kidney Disease/Improving Global Outcomes (KDIGO) guidelines. ${ }^{[12]}$

Patients underwent screening for secondary causes of hypertension based on history, examination and investigations. BP was recorded at the screening and follow-up visits and the changes in systolic BP and diastolic BP were recorded. BP was measured using a Spacelabs automated blood pressure machine, measuring blood pressure at 2-minute intervals. At screening this was done over a 2-hour period and at follow-up over 20 minutes. An average of the last five BP readings was recorded for both screening and follow-up visits. Controlled BP was defined as a blood pressure of $\leq 140 / 90 \mathrm{mmHg}$. Apparent treatment-resistant hypertension was defined as uncontrolled BP while using three or more antihypertensive medications, including $25 \mathrm{mg}$ hydrochlorothiazide daily.

Patients with normal screening blood pressures were referred back to their source healthcare facility.

\section{Statistical analysis}

Statistical analysis was performed using Microsoft Excel 2010 (Microsoft, USA). Two-group comparison was done to investigate for statistical differences between controlled and uncontrolled patients, and between patients with primary hypertension and those with secondary causes. The Welch $t$-test for unpooled variance was used for numerical data to minimise sample size discrepancy and non-
Gaussian distribution. Fisher's exact test was used for categorical data. A level of significance of $p<0.05$ was used.

Selection bias was minimised by including all patients who presented for screening, and measurement bias was minimised through use of diagnostic criteria and definitions. There was no interpretation bias owing to the nature of the study.

\section{Ethical considerations}

Patient confidentiality and privacy were upheld for each patient according to the outlines in the Declaration of Helsinki.

\section{Results}

One hundred and seventy-five patients were enrolled in the study (72 males and 103 females, mean age 46.5 years, of whom $27.6 \%$ were black African, $2.9 \%$ white and $69.1 \%$ of mixed ancestry). Of 18 patients who were not on any antihypertensive treatment, 6 had a normal BP at initial assessment, translating into a prevalence of white-coat hypertension of $3.4 \%$. Of the 175 patients enrolled, 29 (16.6\%) had a normal BP at screening and were discharged back to the referring clinic, $36(20.5 \%)$ with a high BP at screening were lost to follow-up, and $110(62.9 \%)$ with a high BP were evaluated and followed up at the tertiary-level hypertension clinic (Fig. 1). Only

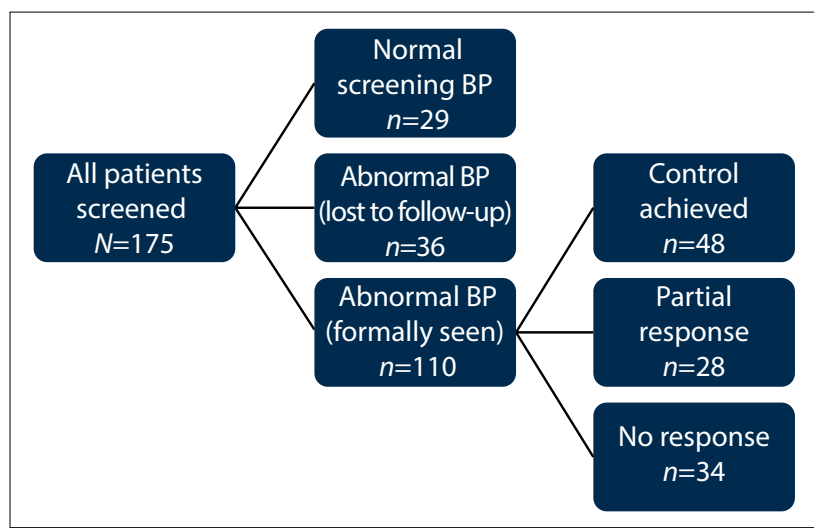

Fig. 1. Flow chart of the patient sample according to screening $B P$ and $B P$ control at follow-up.

Table 1. Gender, age, BMI, BP, LVH and proteinuria in the total patient sample and five patient subgroups

\begin{tabular}{lllllll}
\hline & $\begin{array}{l}\text { All patients } \\
(\mathbf{N = 1 7 5 )}\end{array}$ & $\begin{array}{l}\text { Normal } \\
\text { screening BP } \\
(\boldsymbol{n}=\mathbf{2 9})\end{array}$ & $\begin{array}{l}\text { Controlled } \\
\text { after follow- } \\
\text { up }(\boldsymbol{n}=\mathbf{4 8})\end{array}$ & $\begin{array}{l}\text { Uncontrolled } \\
\text { after follow-up } \\
(\boldsymbol{n}=\mathbf{6 2})\end{array}$ & $\begin{array}{l}\text { Primary } \\
\text { hypertension } \\
(\boldsymbol{n}=\mathbf{1 2 3})\end{array}$ & $\begin{array}{l}\text { Secondary } \\
\text { hypertension } \\
(\boldsymbol{n}=\mathbf{2 3})\end{array}$ \\
\hline Males, $n$ & 72 & 15 & 11 & 27 & 63 & 9 \\
Females, $n$ & 103 & 14 & 37 & 35 & 89 & 14 \\
Age (years), mean (SD) & $46.5(15.6)$ & $40.6(15.4)$ & $46.5(16.9)$ & $49.4(14.7)$ & $45.8(15.3)$ & $51.1(17.2)$ \\
BMI (kg/m²), mean (SD) & $31.0(7.2)$ & $30.8(7.2)$ & $33.4(8.6)$ & $29.6(6.0)$ & $31.3(7.4)$ & $29.2(5.6)$ \\
Screening SBP (mmHg), mean (SD) & $148.7(25.8)$ & $124.0(9.8)$ & $138.4(16.7)$ & $160.4(27.2)$ & $147.1(24.8)$ & $159.7(29.9)$ \\
Follow-up SBP (mmHg), mean (SD) & NA & NA & $125.2(9.2)$ & $159.9(20.3)$ & $143.6(22.3)$ & $152.2(31.7)$ \\
Change in SBP (mmHg), mean (SD) & NA & NA & $-13.2(18.1)$ & $-0.5(27.0)$ & $-6.2(23.1)$ & $-4.9(31.4)$ \\
Screening DBP (mmHg), mean (SD) & $89.3(15.2)$ & $77.2(8.0)$ & $84.6(12.7)$ & $94.1(15.6)$ & $89.1(14.7)$ & $90.9(18.5)$ \\
Follow-up DBP (mmHg), mean (SD) & NA & NA & $76.4(7.9)$ & $94.7(14.1)$ & $86.4(15.2)$ & $88.7(12.9)$ \\
Change in DBP (mmHg), mean (SD) & NA & NA & $-3.8(13.1)$ & $0.6(17.9)$ & $-3.5(16.7)$ & $-1.1(15.7)$ \\
LVH, $n$ (\%) & $86(49.1)$ & $18(62.1)$ & $20(41.7)$ & $31(50.0)$ & $78(51.3)$ & $8(34.8)$ \\
Microalbuminuria, $n$ (\%) & $32(18.3)$ & $0(0)$ & $8(16.7)$ & $19(30.6)$ & $27(17.8)$ & $5(21.7)$
\end{tabular}


48 (43.6\%) of the 110 patients followed up achieved BP control. In terms of TOD, $49.1 \%$ of patients had evidence of LVH and $18.3 \%$ had microalbuminuria.

We divided the patients into five groups, viz. normotensive at screening, controlled BP at follow-up, uncontrolled BP at follow-up, primary hypertension and secondary hypertension. We analysed these five groups in terms of gender, age, BMI, screening and followup diastolic and systolic BP, change in BP, and incidence of LVH and proteinuria (Table 1). We further compared the groups listed below.

\section{Controlled BP v. uncontrolled BP}

In the uncontrolled BP group, 22 of 62 patients had a screening systolic $\mathrm{BP}>160 \mathrm{mmHg}$. In comparison, the screening systolic BP $(p<0.001)$ and diastolic BP $(p<0.001)$ were significantly lower in the controlled group of patients. The BMI was significantly different between the two groups $(p=0.01$ ), being unexpectedly lower in the uncontrolled BP group $\left(29.6 \mathrm{~kg} / \mathrm{m}^{2}\right)$ than in the controlled BP group $\left(33.4 \mathrm{~kg} / \mathrm{m}^{2}\right)$. Gender $(p=0.01)$, potassium level $(p=0.02)$ and microalbuminuria $(p=0.04)$ differed significantly between the controlled and uncontrolled BP groups, with more males, more microalbuminuria and higher potassium levels in the uncontrolled BP group.

No differences were found in age $(p=0.33)$, glucose $(p=0.44)$, total cholesterol $(p=0.83)$, sodium $(p=0.89)$ or uric acid $(p=0.83)$ levels, ethnic distribution $(p=0.08)$, the proportion of patients with LVH $(p=0.11)$ or the proportion of those with secondary causes of hypertension $(p=0.20)$.

\section{Primary v. secondary hypertension}

In total, 23 patients (13.1\%) were diagnosed with secondary hypertension. Of these patients, 2 had normal screening blood pressures and 5 with hypertension were lost to follow-up. The following causes for secondary hypertension were found: $\mathrm{ENaC}$ mutations (34.8\%), primary hyperaldosteronism $(21.7 \%)$, chronic kidney disease $(13.0 \%)$, renovascular disease $(13.0 \%)$, thyroid disease $(8.7 \%)$, aortic coarctation (4.3\%), and phaeochromocytoma (4.3\%).

The screening systolic BP was significantly lower in the group with primary hypertension than in the group with secondary hypertension $(p=0.03)$, but the diastolic BP was not $(p=0.33)$. Age $(p=0.09)$, BMI $(p=0.06)$, and glucose $(p=0.42)$, total cholesterol $(p=0.46)$, sodium $(p=0.30)$, potassium $(p=0.84)$ and uric acid $(p=0.60)$ levels were also not significantly different. No differences were found in gender $(p=0.99)$ or the proportions of patients with LVH $(p=0.18)$, microalbuminuria $(p=0.58)$ or BP control $(p=0.20)$.

\section{White-coat and resistant hypertension}

Of the 175 patients enrolled, 6 not on antihypertensive treatment and 23 on antihypertensive treatment had normal BPs at screening. This translates to estimated prevalences of white-coat hypertension and white-coat effect (higher office BP in hypertensive patients) of 3.4\% and $13.1 \%$, respectively (overall white-coat effect $16.5 \%$ ). Twenty-two (12.6\%) of 175 patients fulfilled the criteria for resistant hypertension at the initial assessment. Of these patients, 7 were lost to further follow-up and 15 were followed up, of whom only 4 (26.6\%) achieved BP control after the 12-month follow-up period.

\section{Discussion}

Hypertension is a common healthcare challenge in SA, and the majority of hypertensive patients can be managed at a primary or secondary healthcare level. In our study, 175 patients were enrolled, all of whom had been referred for tertiary-level management owing to inadequate BP control. Of the patients $16.5 \%$ were found to have a normal $\mathrm{BP}$ at screening, suggesting an underlying white-coat effect, although we cannot exclude the possibility that $13.1 \%$ of these patients who were on antihypertensive treatment improved their adherence prior to the initial assessment. Bhatt et al. ${ }^{[13]}$ showed that 43 of 130 patients were incorrectly diagnosed with apparent resistant hypertension when BP measurements obtained by triage staff were compared with those obtained by trained physicians using a validated $\mathrm{BP}$ device on the same day. Interestingly, $62.2 \%$ of our patients with normal screening BP had evidence of LVH on the ECG, suggesting better adherence prior to assessment or that the white-coat effect may not be completely benign. A recent meta-analysis by Briasoulis et al. ${ }^{[14]}$ concluded that white-coat hypertension is associated with increased cardiovascular morbidity and mortality when compared with normotensive patients followed up over an 8-year period. ${ }^{[14]}$

A large proportion of patients (20.6\%) were lost to follow-up after screening. These patients all warranted further investigations and management, but did not attend the follow-up visits for unidentified reasons. Patient non-adherence to clinic appointments is an important healthcare challenge faced by all clinical disciplines. In a 2010 Cochrane review, ${ }^{[15]}$ the authors comment that appointment reminder systems increased the proportion of individuals who attended for follow-up (odds ratio (OR) $0.41,95 \%$ confidence interval (CI) $0.32-0.51$ ), and in two small trials these also led to improved blood pressure control, the OR favouring intervention (OR 0.54, 95\% CI $0.41-0.73$ ). In the SMS-Text Adherence Support (StAR) study ${ }^{[16]}$ conducted in primary healthcare clinics in Cape Town, an SMS reminder system resulted in only a minor improvement in BP (2 mmHg systolic BP), suggesting that other strategies to improve adherence are required.

Among the $62.9 \%$ of patients who were formally followed up at the specialist clinic, a $43.6 \%$ rate of BP control was attained after a median follow-up period of 232 days. This implies that there is a large proportion of patients $(56.4 \%$ of those referred for specialist intervention) who remain uncontrolled despite specialist management. Encouragingly, specialist intervention in the sample group resulted in mean drops of $13.2 \mathrm{mmHg}$ (range 7.9 - 18.4) in systolic BP and $3.8 \mathrm{mmHg}(4.4-12.0)$ in diastolic BP. Even in the uncontrolled group, there were improvements in $\mathrm{BP}$, which are beneficial. A recent assessment of drug levels for amlodipine and angiotensin-converting enzyme inhibitors in our hypertension clinic suggests that $20 \%$ of patients are non-adherent at any one visit. ${ }^{(17)}$ Interventions to improve patient adherence, such as reducing daily doses, appear to be effective, although there is less evidence of an effect on BP reduction. ${ }^{[18]}$

There was a high prevalence of TOD in our patient sample, with $49.1 \%$ having evidence of $\mathrm{LVH}$ and $18.3 \%$ having microalbuminuria. A recent systematic review of 40444 treated and untreated hypertensive patients found the prevalence of LVH on ECG to be $18 \%{ }^{[19]}$ Our average prevalence of $49.1 \%$ is far higher, suggesting either that our population is inherently at higher risk of LVH or, more plausibly, that there are a greater number of patients with undetected and uncontrolled hypertension in our population. National hypertension work groups and guidelines have advocated for a long time that more resources be allocated to the early detection and management of hypertension in SA communities to prevent the onset of TOD. This would logically translate into significant cost savings for the state, in terms of less expenditure on renal replacement programmes, stroke care and interventional cardiology for ischaemic heart disease. However, this remains a major healthcare challenge, and will necessitate application of greater impetus to hypertension screening and education programmes across all communities.

Our study also aimed to identify key differences between patients who attained BP control and those who did not. In comparison with 
the controlled group, the uncontrolled group was older, comprised more males, had a higher systolic and diastolic BP at screening, and comprised more patients with secondary causes and TOD.

Contrary to current thinking, we found that a low BMI was associated with poor BP control. We know from large Asian and African cohorts ${ }^{[20]}$ that $\mathrm{BP}$ has a non-linear relationship to BMI in females and that there is a cut-off of $21 \mathrm{~kg} / \mathrm{m}^{2}$ below which the relationship is unclear. However, this phenomenon is not found in males and is therefore difficult to explain in our patients. A higher serum potassium level was also found in the uncontrolled hypertension group compared with the controlled group ( $4.3 \mathrm{mmol} / \mathrm{L}$ v. $4.0 \mathrm{mmol} / \mathrm{L})$, but the difference is small and may just be a chance finding.

Our study also looked at the number of patients diagnosed with secondary hypertension. Of the 175 patients referred to our hypertension clinic, $13.1 \%$ were found to have secondary causes of hypertension. This is higher than the traditionally quoted prevalence from community-based studies and may be explained by the selective nature of our sample. We also included $\mathrm{ENaC}$ mutations as a secondary cause, and this test would not have been routinely performed in other studies. When compared with patients with primary or essential hypertension, the patients with secondary hypertension were older and had higher screening and follow-up systolic and diastolic BPs.

The R563Q $\beta$-ENaC mutation and primary hyperaldosteronism together were responsible for $>50 \%$ of cases of secondary hypertension in our study. This reaffirms the high prevalence of both these conditions found in previous studies at our hypertension clinic. ${ }^{[10,21]}$ The R563Q $\beta$-ENaC mutation and primary hyperaldosteronism are currently treated with good success with amiloride and spironolactone, respectively, emphasising the importance of referring patients with apparent treatment-resistant hypertension to a specialist hypertension clinic.

The estimated prevalence of resistant hypertension in our study was $15 \%$. This is lower than the prevalence of resistant hypertension of between $25 \%$ and $35 \%$ from large clinical studies in other countries, ${ }^{[22-24]}$ but higher than the $8.2 \%$ prevalence detected in a recent Italian study of 1177 patients. ${ }^{[25]}$ However, only 4 of the 15 resistant patients followed up in our study achieved BP control, suggesting that these patients are challenging to treat.

\section{Study limitations}

Limitations to the study include its descriptive nature, the assumption that patients are completely adherent to treatment, and the large difference in the sizes of the primary and secondary hypertension comparator groups.

\section{Conclusion}

Hypertension is a major contributor to the disease burden in SA, and not all patients are suitable for management at a primary or secondary healthcare level. Referral to a specialist hypertension clinic was found to have a favourable effect on hypertension management, with a $43.6 \%$ control rate in patients previously uncontrolled at referring facilities. However, this level of control is not ideal. Significant challenges therefore remain, and new strategies need to be developed to deal with them. There are very few specialist hypertension clinics in SA, and these tend to be under-resourced. Further research is required to determine whether specialist intervention in poorly controlled hypertensive patients improves long-term outcome. Another positive finding of this study is that the use of prescreening automated BP monitoring at our hypertension clinic reduced the need for full evaluation and follow-up in $16.5 \%$ of referred patients. Both resistant hypertension and secondary hypertension are prevalent in patients with poor BP control referred to a specialist hypertension clinic. The rate of complications of inadequate BP control in terms of TOD was found to be high, so we recommend that patients with apparent resistant hypertension be referred timeously to tertiary centres for specialist intervention.

\section{References}

1. Lawes CMM, Vander Hoorn S, Law MR, et al. Comparative Quantification of Health Risks: Global and Regional Burden of Disease Attributable to Selected Major Risk Factors. Geneva: World Health Organization, 2004:281-390.

2. World Health Organization. Global Health Risks: Mortality and Burden of Disease Attributable to Selected Major Risks. Geneva: WHO, 2009.

3. Norman R, Gaziano T, Laubscher R, et al. Estimating the burden of high blood pressure in South Africa in 2000. S Afr Med J 2007;97(8):692-698.

4. Steyn K, Gaziano TA, Bradshaw D, et al. Hypertension in South African adults: Results from the 4. Steyn K, Gaziano TA, Bradshaw D, et al. Hypertension in South African
Demographic and Health Survey, 1998. J Hypertens 2001;19(10):1717-1725.

5. South Africa Labour and Development Research Unit, School of Economics, University of Cape Town. 5. South Africa Labour and Development Research Unit, School of Economics, University of Cape Town.
National Income Dynamics Study 2010. Cape Town: South Africa Labour and Development Research Unit, UCT, 2010.

6. Lloyd-Sherlock P, Beard J, Minicuc N, Ebrahim S, Chatterii S. Hypertension among older adults in low and middle-income countries: Prevalence, awareness and control. Int J Epidemiol 2014;43(1):116-128. DOI:10.1093/ije/dyt215

7. Egan BM, Li J, Small J, Nietert PJ, Sinopoli A. The growing gap in hypertension control between insured and uninsured adults. Hypertension 2014;64(5):997-1004. DOI:10.1161/ HYPERTENSIONAHA. 114.04276

8. Heimark S, Eskas PA, Mariampillai JE, Larstorp AC, Hoieggen A, Fadl Elmula FE. Tertiary work-up of apparent treatment-resistant hypertension. Blood Press 2016;Apr 28:1-7. [Epub ahead of print] DOI: .

2014;25(6):288-294. DOI:10.5830/CVJA-2014-062

10. Rayner BL Owen EP, King JA, et al A new mutation, 25630 of the beta subunit of the epithelinl Rayner BL, Owen EP, King JA, et al. A new mutation, R563Q, of the beta subunit of the epithelial
sodium channel associated with low-renin, low-aldosterone hypertension. J Hypertens 2003;21(5):921926. DOI:10.1097/01.hih. $0000059009.82022 .9 \mathrm{~b}$

11. Levey AS, Bosch JP, Lewis JB, Greene T, Rogers N, Roth D. A more accurate method to estimate glomerular filtration rate from serum creatinine: A new prediction equation. Modification of Diet in Renal Disease Study Group. Ann Intern Med1999;130(6):461-470.

12. KDIGO 2012. Clinical practice guideline for the evaluation and management of chronic kidney disease. Kidney Int Suppl 2013;3(1):5-14. DOI:10.1038/kisup.2012.64

13. Bhatt H, Siddiqui M, Judd E, Oparil S, Calhoun D. Prevalence of pseudoresistant hypertension due to inaccurate blood pressure measurement. J Am Soc Hypertens 2016;10(6):493-499. DOI:10.1016/j. jash.2016.03.186

14. Briasoulis A, Androulakis E, Palia M, Papageorgiou N, Tousoulis D. White-coat hypertension and cardiovascular events: A meta-analysis. J Hypertens 2016;34(4):593-599. DOI:10.1097/ HJH.0000000000000832

15. Glynn LG, Murphy AW, Smith SM, Schroeder K, Fahey T. Interventions used to improve control of blood pressure in patients with hypertension. Cochrane Database Syst Rev 2010, Issue 3. Art. No: CD005182. DOI:10.1002/14651858.CD005182.pub4

16. Bobrow K, Farmer AJ, Springer D, et al. Mobile phone text messages to support treatment adherence in adults with high blood pressure (SMS-Text Adherence Support [StAR]): A single-blind, randomized trial. Circulation 2016;133(6):592-600. DOI:10.1161/CIRCULATIONAHA.115.017530

17. Jones E, Decloedt E, Blockman M, et al. Assessing antihypertensive adherence with therapeutic drug monitoring. J Hypertens 2015;33(eSuppl 1):e280. DOI:10.1097/01.hjh.0000468220.61883.fe

18. Schroeder K, Fahey T, Ebrahim S. Interventions for improving adherence to treatment in patients with high blood pressure in ambulatory settings. Cochrane Database Syst Rev 2004, Issue 3. Art. No: CD004804. DOI:10.1002/14651858.CD004804

19. Cuspidi C, Rescaldani M, Sala C, Negri F, Grassi G, Mancia G. Prevalence of electrocardiographic left ventricular hypertrophy in human hypertension: An updated review. J Hypertens 2012;30(11):20662073. DOI:10.1097/HJH.0b013e32835726a3

20. Kaufman JS, Asuzu MC, Mufunda J, et al. Relationship between blood pressure and body mass index in lean populations. Hypertension 1997;30(6):1511-1516. DOI:10.1161/01.HYP.30.6.1511

21. Jones ESW, Owen EP, Davidson JS, van der Merwe L, Rayner BL. The R563Q mutation of the epithelial sodium channel beta-subunit is associated with hypertension. Cardiovasc J Afr 2011;22(5):241-244. Dodium channel beta-subunit

22. Gupta AK, Nasothimiou EG, Chane CL, Sever PS, Dahlof, Poulter NR, on behalf of the ASCOT investigators. Baseline predictors of resistant hypertension in the Anglo-Scandinavian Cardiac Outcome Trial (ASCOT): A risk score to identify those at high-risk. J Hypertens 2011;29(10):20042013. DOI: 10.1097/HJH.0b013e32834a8a42

23. Cushman WC, Ford CE, Cutler JA, et al., for the ALLHAT Collaborative Research Group. Success and predictors of blood pressure control in diverse North American settings: The Antihypertensive and Lipid-Lowering and Treatment to Prevent Heart Attack Trial (ALLHAT). J Clin Hypertens 2002;4(6):393-404. DOI:10.1111/j.1524-6175.2002.02045.x

24. Jamerson K, Weber MA, Bakris GL, et al., for the ACCOMPLISH trial investigators. Benazepril plus amlodipine or hydrochlorthiazide for hypertension in high-risk patients. N Engl J Med 2008;359(23):2417-2428. DOI:10.1056/NEJMoa0806182

25. Galletti F, Barbato A. Prevalence and determinants of resistant hypertension in a sample of patients followed in Italian hypertension centers: Results from the MINISAL-SIIA study program. J Hum Hypertens 2016;130(5):453-461. DOI:10.1017/S0022215116000761 